\title{
Diamond Monochromator for High Heat Flux Synchrotron X-ray Beams.*
}

\author{
A.M. Khounsary, R.K. Smither and S. Davey \\ Advanced Photon Source \\ Argonne National Laboratory \\ 9700 South Cass Avenue \\ Argonne, IL 60439
}

December, 1992

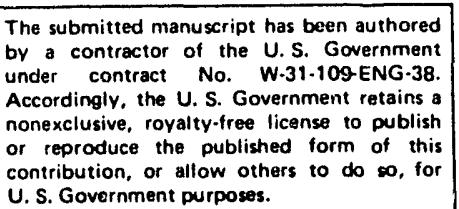

\section{DISCLAIMER}

This report was prepared as an account of work sponsored by an agency of the United States Government. Neither the United States Government nor any agency thereof, nor any of their employees, makes any warranty, express or implied, or assumes any legal liability or responsibilit, $r$ the accuracy, completeness, or usefulness of any information, apparatus, product, or process disclosed, or represents that its use would not infringe privately owned rights. Reference herein to any specific commercial product, process, or service by trade name, trademark, manufacturer, or otherwise does not necessarily constitute or imply its endorsement, recommendation, or favoring by the United States Government or any agency thereof. The views and opinions of authors expressed herein do not necessarily state or reflect those of the United States Government or any agency thereof. 


\title{
Diamond Monochromator for High Heat Flux Synchrotron X-ray Beams
}

\author{
A.M. Khounsary, R.K. Smither, and S. Davey, \\ Advanced Photon Source \\ A. Purohit, Engineering Physics Division \\ Argonne National Laboratory \\ Argonne, IL 60439
}

\begin{abstract}
Single crystal silicon has been the material of choice for $\mathrm{x}$-ray monochromators for the past several decades. However, the need for suitable monochromators to handle the high heat load of the next generation synchrotron $x$-ray beams on the one hand and the rapid and on-going advances in synthetic diamond technology on the other make a compelling case for the consideration of a diamond monochromator system. In this paper, we consider various aspects, advantages and disadvantages, and promises and piffalls of such a system and evaluate the comparative performance of a diamond monochromator subjected to the high heat load of the most powerful x-ray beam that will become available in the next few years. The results of experiments performed to evaluate the diffraction properties of a currently available synthetic single crystal diamond are also presented. Fabrication of a diamond-based monochromator is within present technical means.
\end{abstract}

\section{INTRODUCTION}

The combination of high power and high power density associated with the $\mathrm{x}$-ray beams generated by insertion devices at the third generation synchrotron radiation facilities has created new challenges in the design of the beamline components that intercept the $\mathrm{x}$-ray beams. The challenge is nowhere greater than in the design of the first optical components, notably monochromators and mirrors, which must provide acceptable performance under the thermal load of the $\mathrm{x}$-ray beams.

A resurgence of research activities in the development of monochromators for high heat load beamlines has led to examination of many aspects of the problem. Much attention has been concentrated on the cooling of monochromators with the aim to reduce the temperature gradient and thus the thermal distortion in the system. ${ }^{1}$ Novel monochromator designs such as the inclined ${ }^{2-3}$ or asymmetric ${ }^{4}$ monochromator have also been suggested. The combined effort has resulted in the design of monochromators that can adequately handle the needs of the synchrotron community for the near future. For the $x$-ray beams that come on line in the second half of the decade, the currently available designs may not be adequate.

One area with substantial potential in the design of high performance monochromators is the monochromator material selection. In this paper, we consider this aspect of the problem and specifically suggest diamond as a material of choice. While diamond has previously been used in diffraction work, in this paper we consider its application in high heat load monochromators and provide a preliminary study of the design, fabrication, analysis, and characterization aspects of a diamond monochromator system.

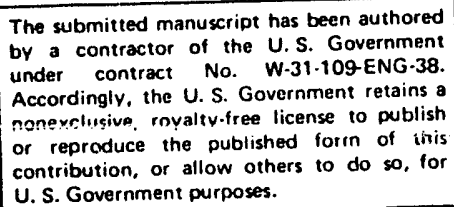




\section{MONOCHROMATOR MATERIAL}

Currently, silicon is almost universally used in the design of monochromator systems for high energy (over 2-3 keV) x-ray beams. The choice arises from the availability of low-cost, large-size, and extremely high quality single crystal silicon developed primarily for the semiconductor industry. Single crystal diamonds have not been used for this application for a number of reasons. The two main (and mutually reinforcing) reasons have been a general lack of need for, and unavailability of, suitable single crystal diamonds for $\mathrm{x}$-ray optics applications.

There are now some compelling reasons why the subject of a diamond monochromator should be reassessed. To begin with, the $\mathrm{x}$-ray beams produced by undulators at modern synchrotron facilities are so intense that they cause unacceptably high thermal distortion in the cooled optical elements on which they impinge. Thus, an optically suitable single crystal with high thermal conductivity and low thermal expansion coefficient is highly desirable. Single crystal diamond at room temperature fits this description rather well as does silicon at cryogenic temperatures. 5,6

At temperatures below $20 \mathrm{~K}$, single crystal silicon has a negligible thermal expansion coefficient, and its thermal conductivity is about $50 \mathrm{~W} / \mathrm{cm}-\mathrm{K}$ (i.e., about 12 times better than that of copper at room temperature). 5 It has a negative thermal expansion coefficient below $125 \mathrm{~K}$ (i.e., it contracts upon heating). The thermal expansion coefficient is zero at about $125 \mathrm{~K}$ where the thermal conductivity is about $6 \mathrm{~W} / \mathrm{cm}-\mathrm{K}$ (or $50 \%$ better than that of copper at room temperature). Thus, a cryogenically cooled silicon monochromator system, operated for example at liquid nitrogen temperature, is conceptually attractive. In practice, however, the design of a system capable of removing several $\mathrm{kW}$ of heat with on-the-surface peak heat fluxes in excess of $50 \mathrm{~W} / \mathrm{mm}^{2}$ (expected from APS Undulator A, for example) is a rather formidable task, the most obvious complications of which are the critical heat flux issue, the required large cooling area that necessitates a multi-layer heat exchanger, and a robust design for maintaining the integrity and the figure under thermal cycling. It is more realistic to consider a cryogenically cooled silicon monochromator system with an inclined or asymmetric geometry in which the incident heat flux is spread out over a much larger area than in a conventional monochromator. ${ }^{2}$

Diamond at room temperature offers a more manageable and yet competitive alternative to silicon at cryogenic temperatures. Single crystal diamond can have a thermal conductivity in excess of $21 \mathrm{~W} / \mathrm{cm}-\mathrm{K}$. This is five times better than copper at room temperature, and the ratio is accentuated at lower temperatures, to some 25 times at liquid air temperature (Type IIa diamond has a fantastic thermal conductivity of about $100 \mathrm{~W} / \mathrm{cm}-\mathrm{K}$ at about $100 \mathrm{~K}$ ). Thus, it is apparent that, at least from a thermal point of view, a diamond-based monochromator system can provide an option for dealing with high thermal load $\mathrm{x}$-ray beams.

In using diamond for the design of an z-ray monochromator system, however, a number of issues must first be resolved. These concern the a /ailability, quality, and suitability of diamonds for synchrotron applications. These issues are discussed next.

\section{SINGLE CRYSTAL DIAMONDS}

Diamonds 6-9 are classified (based on their IR and UV absorptions) as Type I or II depending on whether nitrogen, a common impurity, is present or not. Each Type is further subdivided into $a$ or $b$, to indicate the specific form in which impurities are present. A majority of natural diamonds are Type I, with high concentrations of nitrogen, while Type II diamonds (which make up about $2 \%$ of diamonds) have 
little or no nitrogen. Type II diamonds contain impurities with concentrations of about 10 parts per million and as gem stones are considered to be nearly "perfect" and free from "defects." Most of these are Type IIa, having a high electrical resistivity $\left(-5 \times 10^{14} \mathrm{ohm}-\mathrm{m}\right)$, and are essentially insulators. Type Ilb diamonds, on the other hand, are semiconductors with a resistivity of $100 \mathrm{ohm}-\mathrm{m}$ or less, due to the presence of boron as an impurity.

High quality natural diamonds are available in sizes up to $10 \times 10 \times 1 \mathrm{~mm}^{3}$ or larger. ${ }^{10-11}$ The price 10 for a $10 \times 10 \times 0.25 \mathrm{~mm}^{3}$ Type. Ila diamond is under $\$ 8000$. Larger area diamonds can be available at substantially higher costs. Type Ilb crystal prices are about twice those of Type Ila diamonds. Type Ia diamonds are not suitable for the present application, while Type Ib diamonds, which are available in sizes up to $6 \mathrm{~mm} \times 6 \mathrm{~mm} \times 0.25 \mathrm{~mm}$ (or thicker), are priced at about $\$ 2000$ or roughly about $20 \%$ higher than Type IIa diamonds of the same size. 10

For x-ray diffraction applications, one may ideally want to use a perfect diamond crystal, that is a diamond free of all impurities and lattice defects \& dislocations. Such crystals are rare, and extensive testing of many crystals is necessary to select a suitable specimen. We note, however, that certain imperfections in single crystal diamonds may even be desirable in certain $\mathbf{x}$-ray diffraction applications.

A high quality diamond, in the aesthetic sense, with no "impurities" may still be (and often is) imperfect, in the diffraction sense. This is due to lattice defects and lattice deformations. The former include missing or displaced atoms from the points of their geometric location and local elastic deformations which alter the inter atomic spacing and the bond lengths Lattice deformations are due to the presence of a few or a large number of elastically stained regions in the crystal leading to a crystal that is divided into many small regions with slightly different lattice orientations. This is the mosaicity of the single crystal diamond that broadens the rocking curve.

Natural diamonds are generally stressed. Sometimes a part of this stress may be annealed by heating the diamond in vacuum (to prevent oxidation) or by heating it to very high temperatures for a short period of time (to prevent graphitization.) in an inert environment

A rather unexpected result of a limited number of studies on the diffraction properties of natural diamonds is that because of a type of dislocation commonly found in and characteristic of the more "perfect" Type IIa diamonds, Type Ib diamonds are actually mcie suitable for x-ray monochromators. ${ }^{12}$ To our knowledge, the only reported confirmation of this by precise rocking curve measurements is due to Jackson 13 who obtained a double crystal rocking curve width (apparently the full width at half maximum, or FWHM) of about 150 arc seconds for a Type IIa diamond and only 10 arc seconds for a Type Ib diamond, both from (022) crystal planes. The photon energy is not specified, but the theoretical FWHM of the double crystal rocking curve calculated 14 for these specimen ranges from 1.5 arc seconds for 20 $\mathrm{keV}$ to 32 arc seconds for $5 \mathrm{keV}$ photons. Jackson 13 also notes that the annealing of the Type Ila crystals to $1000^{\circ} \mathrm{C}$ failed to affect the rocking curve width.

Because of the recent availability of synthetic single crystal diamonds and our belief that the rapid advances in diamond technology may soon result in larger area diamonds of high consistency and quality, we have examined Type Ib synthetic crystals produced by Sumitomo Electric. 15 These crystals known as Sumicrystals $^{\mathrm{TM}}$, are about $5 \times 5 \times 0.3 \mathrm{~mm}^{3}$ in size and are developed primarily as a heat sink material. They have a yellow color indicative in nitrogen impurity, which is reported by the manufacturer to be on the order of tens of parts per million. The crystals are cut, using a Yag laser beam, from larger crystals that are synthesized in a high pressure (about 50,000 atmospheres) and high temperature (over $1300^{\circ} \mathrm{C}$ ) process. The Sumicrystals ${ }^{\mathrm{TM}}$ have not, to our knowledge, been adequately characterized for $\mathrm{x}$-ray 
applications, although there are indications that they may be better than most natural diamonds in crystalline quality and in consistency.

The high pressure-high temperature technique to produce synthetic diamond crystals (first announced by the General Electric (GE) researchers in 1955 and detailed later, ${ }^{16}$ ) has been modified and refined to produce larger and higher quality single crystal diamonds. The only single crystal diamonds that General Electric now supplies are the isotropically pure $(99.99 \%) \mathrm{C}^{12}$ crystals. 17 Owing to the much reduced irregularities in the crystal lattice vibrations present in diamonds with natural isotropic composition, the GE crystals have a room temperature thermal conductivity of $33 \mathrm{~W} / \mathrm{cm}^{2}-\mathrm{K}$, or $50 \%$ above that of the best natural diamonds. Two (400) GE samples ( $4 \mathrm{~mm} \times 4 \mathrm{~mm}$ in area) evaluated on the $\mathrm{X}-25$ beamline at Brookhaven National Laboratory 18 using a (440) silicon analyzer gave rocking curve FWHMs of 2.5 and 8 arc seconds, confirming that at least one of the two is an extremely good crystal. Other investigations show that the rocking curve widths for these crystals are not perceptively dependent on the isotropic composition. 19 The size of GE crystals, according to the supplier, does not exceed $5 \mathrm{~mm} \times 5 \mathrm{~mm}$ in area. 17

During the course of the present study, it has become obvious that the potential $x$-ray optical applications of synthetic diamonds have not been communicated to the respective manufactures. In fact, the Sumicrystals ${ }^{\mathrm{TM}}$ diamonds that we have examined are mostly used for heat sink applications, and have not benefited from any special handling (in cutting, polishing, etc.) appropriate for optical applications. We have measured the RMS surface roughness of these diamond crystals to be about $10 \AA$ with a radius of curvature of about $10 \mathrm{~m}$. The largest synthetic diamond crystals currently available 15 from Sumitomo is about $12 \times 12 \times 0.3 \mathrm{~mm}^{3}$ and costs about $\$ 32,000$. An $8 \times 12 \times 0.3 \mathrm{~mm}^{3}$ will cost about $\$ 19,000$. As we shall shortly see, these sizes are adequate for the present application.

\section{ROCKING CURVE MEASUREMENTS}

Two Sumicrystal ${ }^{\mathrm{TM}}$ diamond specimens, $5 \times 5 \times 0.3 \mathrm{~mm}^{3}$ in size, were first tested using a Laue camera to determine the crystal planes. The large surface area of the crystals were found to be nearly parallel to the (400) planes. These planes were used in the Bragg diffraction experiments to determine the quality of the synthetic crystals. The experimental setup for these experiments is shown in Fig. 1. The radiation from a Mo-K x-ray source (on the right) passes through a set of double (vertical and horizontal) slits and impinges on the first diamond crystal. The diffracted beam from this crystal passes through a second set of double slits and impinges on the second crystal where the radiation is diffracted for a second time. The intensity of this diffracted beam is recorded with the $\mathrm{x}$-ray detector on the left. This is the standard non-dispersive geometry used to determine the average quality of two crystals. The horizontal widths of the first and second slits are $0.025 \mathrm{~mm}$ and $0.15 \mathrm{~mm}$, respectively. These are sufficient to limit the diffraction to one of the $\mathrm{K} x$-ray lines (K-LIII) in the Mo spectrum. The footprint of the beam on the second crystal is about $0.6 \mathrm{~mm}$ horizontally and $3.0 \mathrm{~mm}$ vertically. The double crystal rocking curve (photon count rate as a functior of Bragg angle) is obtained by rotating the second crystal and recording the photon count rate.

The set up shown in Fig. 1 was first tested by measuring the double crystal rocking curve FWHMs of nearly perfect silicon (111) and (333) crystals. Values of 4.8 and 0.96 arc seconds, respectively, were obtained, which when divided by $\sqrt{2}$ give the corresponding average widths of individual crystals as 3.38 and 0.68 arc seconds. These values are accurate to better than $3 \%$ when compared to the theoretical values (Darwin widths) of 3.32 and 0.66 arc seconds, respectively. 
The double crystal rocking curve of diamond (400) crystals was then obtained as shown in Fig. 2. Also shown in Fig. 2 for comparison is the rocking curve obtained by replacing the diamonds with silicon (111) crystals without changing the photon source or the slit sizes. The measured double crystal rocking curve FWHM for diamond (400) is 6.2 are seconds and for silicon (111) is 4.8 are seconds. If the diamond crystals were perfect, one would expect to obtain 14 a rocking curve with a FWHM of 0.97 are seconds as shown in Fig. 3. Thus, most of the measured line width in the diamond is related to the crystal imperfections. The measured average width of a Sumicrystal ${ }^{\mathrm{TM}}$ is then $6.2 / \sqrt{2}=4.4$ arc seconds, while a perfect diamond (400) would have a FWHM (Darwin width) of $0.97 / \sqrt{2}=0.69$ arc seconds.

With the above information, it is possible to estimate the average mosaic width of the diamond crystals. Assuming that the mosaic width adds to the Darwin width as the square root of the sum of the squares, the mosaic width will be given by the square root of the difference between the squares of the measured and theoretical (Darwin) widths, that is,

Mosaic width $=\left\lceil 4.4^{2}-0.69^{2}\right]^{1 / 2}=4.35$ arc seconds

Assuming that the mosaicity is isotropic, one can now use this value of the mosaic width to estimate the FWHM of the these diamond crystals when they are used to diffract $x$-rays, for example, from the (111) planes. One combines the theoretical diamond (400) width of 3.1 arc seconds with the mosaic width of 4.35 arc seconds to obtain the value of 5.3 arc seconds for this width. Thus a two crystal monochromator system using these diamond crystals with the (111) planes should generate a rocking curve with a FWHM of $5.3 \sqrt{2}$, or 7.5 arc seconds. The results of the rocking curve experiments are summarized in Table I.

Table I. Results of the rocking curve experiments with diamond and silicon crystals. All experiments were performed with the Mo K-LIII x-ray. Given widths are all for one crystal.

\begin{tabular}{|l|c|}
\hline \multicolumn{1}{|c|}{ Description } & $\begin{array}{c}\text { width } \\
\text { (arc seconds) }\end{array}$ \\
\hline Measured average width of the synthetic (400) diamond specimens & 4.4 \\
Theoretical (Darwin) width of a perfect (400) diamond crystal & 0.69 \\
Estimated mosaic width of the synthetic diamond specimens & 4.35 \\
Estimated width of the synthetic (111) diamond crystal* & 5.3 \\
\hline
\end{tabular}

*denotes inferred values based on the computed mosaicity.

The comparison of the rocking curves of the diamond (400) and perfect silicon (111) shown in Fig. 2 is of special interest because not only the widths but also the peak intensities are quite similar. If diamond crystals of this quality were used in a two crystal monochromator, both the angular resolution (which is a function of the rocking curve width and the synchrotron beam opening angle) and the peak count rate in the diffracted beam (which is a function of the integrated area under the rocking curve and thus is sensitive to both the height and width if the curve) would be quite similar.

Our limited tests indicate that the synthetic single crystal diamonds produced at this time are already of sufficient quality to make usable double crystal monochromator systems. The main difference between a double crystal monochromator using these (400) diamonds and perfect silicon (111) is that the Bragg angle in the diamond case is a factor of 3.6 larger. This will improve the energy resolution by a similar 
factor since $\Delta \mathrm{E} / \mathrm{E}$ is proportional to $\Delta \theta / \theta$. Here, $\Delta \theta$ is the angular resolution of the monochromator, which is similar for both cases, and $\theta$ is the Bragg angle, which for diamond (40)) is 3.6 times larger than for silicon (111). The integral diffraction efficiency for the (111) planes in diamond is expected to be larger than for the $(4(K))$ planes, so the diamond (111) planes will produce monochromators with even higher efficiencies.

\section{FABRICATION OF A DIAMOND MONOCHROMATOR}

Although single crystal diamonds currently available are rather modest in size, they are adequate for the collimated high power density $\mathrm{x}$-ray beams generated by undulators. A $12 \times 8 \mathrm{~mm}^{2}$ diamond is large enough to intercept the central cone of the APS Undulator A. 2,20 At $30 \mathrm{~m}$ from the source, the central cone of the $\mathrm{x}$-ray beam at closed gap (at which the power loading is maximum) is about $3.6 \mathrm{~mm}$ horizontally and $1.2 \mathrm{~mm}$ vertically (full width at zero height). This means that a (111) diamond crystal, 12 $\mathrm{mm}$ long, can intercept and diffract the entire central cone radiation at Bragg angles of $5.7^{\circ}$ or larger. This covers the entire 4-30 keV tuning range of Undulator A. Small(er) Bragg angles corresponding to high(er) diffracted photon energies require tuning the undulator to higher harmonic energies at which the power loading of the beam is substantially reduced and therefore a silicon monochromator can be used.

Because of its low atomic number, diamond absorbs less $\mathrm{x}$-ray radiation than does silicon of identical thickness. The PHOTON 21 program was used to calculate the absorbed values. The results are shown in Fig. 4 for APS Undulator A (see specifications in Table II). The thickness of the diffracting diamond can be as small as tens of microns. The thinner the diamond, the less the absorbed radiation, and therefore the smaller the thermal load on it. It is thus advantageous to use a thin diamond monochromator if the crystal can be convectively surface-cooled, for example, by a helium or nitrogen jet. If the crystal is edge cooled, then the thinner the crystal, the smaller is the conduction area for the transfer of the heat from the center of the crystal to its cooled periphery, and the net effect on the temperature and strain in the crystal is, in general, insignificant. The important parameter in edge cooling is the effective heat transfer coefficient at the crystal boundaries. Because of the high conductivity of diamond, a doubling of the effective heat transfer coefficient at the crystal edges will reduce the maximum temperature in the crystal nearly by half. Thus, a thin crystal with edge cooling may be an option depending on the absorbed heat load and the edge cooling efficiency. For the APS Undulator A beam considered in this study, we assume that the diffracting single crystal diamond is bonded to a substrate made of polycrystalline diamond to build what we call an integral diamond crystal. Polycrystalline diamonds can have high thermal conductivities 22 approaching that of single crystal diamonds and, more importantly, they can be produced in large sizes using chemical vapor deposition (CVD) or associated techniques. The diamond (or possibly silicon) diffracting element can be bonded 23 to a CVD substrate with appropriately configured cooling channels With a carefully selected bonding procedure, one may be able to produce strain-free diamond to diamond bonding. We are unaware of any work in which the stress levels in such bonding were measured. An alternative technique would involve deposition of CVD diamond directly on the diffracting diamond element. Again, we are unaware of any work to prodsce or test stain-free bonding using this technique but believe that this may not be an insurmountable problem.

\section{THERMAL AND STRUCTURAL ASPECTS OF AN INTEGRAL DIAMOND MONOCHROMATOR}

In order to determine the relative performance of a diamond-based versus a silicon-based monochromator system, the slope errors resulting from the thermal distortion of the monochromators under the high heat load of an x-ray beam are required. As a rule of thumb, a figure of merit for the 
performance is given by the ratio $\mathrm{k} / \alpha$, where $\mathrm{k}$ is the thermal conductivity and $\alpha$ is the thermal expansion coeflicient. As seen from the property data in Table II, single crystal diamond can have a figure of merit 40 to 50 times higher than silicon at room temperature. The thermal conductivity of polycrystalline diamond is in the $7-21 \mathrm{~W} / \mathrm{cm}^{2}-\mathrm{K}$ range (and possibly higher). ${ }^{22}$ Its thermal expansion coefficient is similar to that of single crystal diamond.

Table II. Properties of single crystal diamond and silicon at room temperature. 6-9

\begin{tabular}{|l|l|l|}
\hline Property & Diamond & Silicon \\
\hline Atomic number, $\mathrm{Z}$ & 6 & 14 \\
Density $\left(\mathrm{g}^{\mathrm{c}} \mathrm{cm}^{3}\right)$ & 3.516 & 2.330 \\
Thermal conductivity $(\mathrm{W} / \mathrm{cm}-\mathrm{K})$ & 21 & 1.25 \\
Thermal expansion coefficient $\left(\mathrm{K}^{-1} \times 10^{-6}\right)$ & 0.8 & 2.33 \\
Specific heat $(\mathrm{J} / \mathrm{Kg}-\mathrm{K})$ & 520 & 750 \\
Thermal diffusivity $\left(\mathrm{cm}^{2} / \mathrm{s}\right)$ & 11.5 & 0.72 \\
Young's modules $(\mathrm{GPa})$ & 1,050 & 167 \\
Poisons ratio & $0.1-0.29$ & 0.3 \\
Melting point $\left({ }^{\circ} \mathrm{C}\right)$ & 4300 & 1420 \\
Tensile strength $(\mathrm{GPa})$ & $>3$ & $\mathrm{NA}$ \\
Yield strength $(\mathrm{MPa})$ & $\mathrm{NA}$ & $1240-2060$ \\
Lattice spacing $(\AA)$ & 3.5670 & 5.4305 \\
\hline
\end{tabular}

For a more detailed comparison of the performances, we evaluate the temperature fields and the resulting slope errors in the two monochromator systems, one silicon and the other diamond. The radiation source is assumed to be Undulator A at closed gap $(11.5 \mathrm{~mm})$ on the 7-GeV APS storage ring with a positron current of $100 \mathrm{~mA}$. The total power of the source is $3.8 \mathrm{~kW}$, and the peak normal incidence heat flux at the monochromator $30 \mathrm{~m}$ from the center of the undulator is about $150 \mathrm{~W} / \mathrm{mm}^{2}$. The FWHMs of the beam in the horizontal and vertical directions are 8.2 and $2.9 \mathrm{~mm}$, respectively. The central cone of the beam, which contains most of the desired (harmonic) photons, has a much smaller footprint. For APS Undulator A at $30 \mathrm{~m}$ from the source, the entire (full width at zero height) central cone has a footprint of $3.6 \mathrm{~mm}$ horizontally and $1.2 \mathrm{~mm}$ vertically.

In the computations that follow, it is assumed that an aperture with an opening of $3.6 \mathrm{~mm}$ and $1.8 \mathrm{~mm}$ in the horizontal and vertical directions, respectively, is placed upstream of "ne monochromator. Note that the vertical dimension of this slit is $50 \%$ larger than the $1.2 \mathrm{~mm}$ of the beam central cone.

It is also assumed that the entire heat load intercepted by the monochromator is absorbed on the surface, an assumption that is more appropriate for silicon than for diamond. In fact, as shown in Fig. 4, for APS Undulator A with a characteristic energy of $23.5 \mathrm{keV}$, about $30 \%$ of the power is absorbed in a 1 $\mathrm{mm}$ thick diamond. This figure for silicon is about $50 \%$. In-depth absorption of heat will generally lead to reduced temperatures and strains. As mentioned previously, the low absorption of hard $\mathrm{x}$-rays in diamond favors the possible use of a thin single crystal diamond in Bragg or Laue geometries. For example, for typical incident angles greater than $5^{\circ}$, a $0.2 \mathrm{~mm}$ diamond set to diffract Undulator A beam will absorb no more than $35 \%$ of the incident beam power. Note that the actual beam path length in the $0.2 \mathrm{~mm}$ thick foil is $2.2 \mathrm{~mm}$. It may be possible to edge cool the thin diamond. The cooling, as we have noted, would have to be exceptionally good to maintain moderate temperatures and strains in the diamond. 
In the present study, the total power intercepted by the monochromator through the aforementioned aperture is $860 \mathrm{~W}$. We set the monochromators to diffract third harmonic radiation $(12.6 \mathrm{keV})$ from Undulator $A$ at closed gap. The crystals are assumed to be $1 \mathrm{~cm}$ thick. The widths and lengths are each 2 $\mathrm{cm}$ larger than the corresponding dimensions of the beam footprint. The thickness of $1 \mathrm{~cm}$ is arbitrarily chosen for comparison only, and, in fact, it is neither necessary nor optimal to have such thick substrates. The substrates are assumed to be cooled on the back surface by liquid gallium. The heat transfer coefficient used is $5 \mathrm{~W} / \mathrm{cm}^{2}-\mathrm{K}$. Again, this value is somewhat arbitrary but sufficient for the present comparative study. Table III summarizes these input data.

Table III. Parameters and data used in thermal and structural analyses.

\begin{tabular}{|l|l|}
\hline Parameter & Data \\
\hline Radiation Source & $2.5 \mathrm{~m} \mathrm{Undulator}$ A (closed gap) \\
Beam current & $100 \mathrm{~mA}$ \\
Total power & $3.8 \mathrm{~kW}$ \\
Power density & $135 \mathrm{~kW} / \mathrm{mrad}^{2}$ \\
Beam V-FWHM @30 m & $2.9 \mathrm{~mm}$ \\
Beam H-FWHM @30 m & $8.1 \mathrm{~mm}$ \\
Thermal Slit Location & $30 \mathrm{~m}$ from the source \\
Slit opening $(\mathrm{v}$ h) & $1.8 \times 3.6 \mathrm{~mm}$ \\
Beam footprint $(\mathrm{v} x \mathrm{~h})$ & $7.5 \mathrm{~mm} \times 3.6 \mathrm{~mm}$ \\
Absorbed radiation & surface absorption assumed \\
Monochromator & diamond or silicon \\
Nionochromator location & $30 \mathrm{~m} \mathrm{from} \mathrm{the} \mathrm{source}$ \\
Cooling (on back surface) & gallium \\
Heat transfer coefficient & $5 \mathrm{~W} / \mathrm{cm}{ }^{2}-\mathrm{K}$ \\
Total power intercepted & $860 \mathrm{~W}$ \\
Peak normal incident heat flux & $150 \mathrm{~W} / \mathrm{mm}^{2}$ \\
Diffracting photon Energy & $12.6 \mathrm{keV}$ \\
Undulator harmonic & $3 \mathrm{rd}$ \\
\hline
\end{tabular}

The model used in the thermal-structural analyses of the diamond monochromator is shown in Fig. 5 . The dimensions of the beam footprints and the monochromator components are given in Table III. The thickness of the single crystal diamond (assumed to have been bonded onto the substrate) is $0.5 \mathrm{~mm}$, which is more than suftıcient for diffraction purposes. In the case of silicon, the substrate is also the diffracting element. Because silicon has a relatively low thermal conductivity, we have also considered a silicon crystal, $0.1 \mathrm{~cm}$ thick, to show the effect of reducing substrate thickness.

The temperature profiles along the $\mathrm{AA}^{\prime}$-axis (Fig. $\check{\text { ) }}$ ) for the diamond and silicon monochromators with 1-cm substrates are shown in Fig 6 . In the case of diamond, the temperature on the top surface of the diffracting element (the heavy line) is slightly above the top surface of the substrate (the light line). For the silicon case, they are the same surface and thus the same temperature. The footprint region, which is smaller for the diamond monochromator (larger Bragg angle), is highlighted by the thicker line in Fig. 6 . The maximum temperature rise in the silicon monochromator is about $660^{\circ} \mathrm{C}$, while in the diamond monochromator it is about $55^{\circ} \mathrm{C}$. A reduction in the thickness of the silicon monochromator from $1.0 \mathrm{~cm}$ to $0.1 \mathrm{~cm}$ will not li wer the temperatures substantially (see Table IV). 
Figure 7 shows the corresponding thermal distortions for the two monochromators. It plots the displacement in the plane of scattering along the length (A- $A^{\prime}$ in Fig. 5) of the crystal monochromators. The maximum displacements for silicon and diamond crystals are $0.27 \mu \mathrm{m}$ and $14 \mu \mathrm{m}$, respectively. The displacement in a $0.1-\mathrm{cm}$-thick silicon crystal is not significantly different from that in a 1.0 -cm-thick crystal. It should be noted, however, that this displacement would be substantially less if (as is normally the case) the crystal were restrained. The maximum temperature in the crystal is reduced by more efficient cooling, while the temperature gradient across the thickness, to a first approximation, remains unaffected.

Table IV. Simulation data and results for diamond and silicon monochromators.

\begin{tabular}{|c|c|c|c|}
\hline Material & Diamond & \multicolumn{2}{|c|}{ Silicon } \\
\hline Diffracting planes & $(111)$ & \multicolumn{2}{|c|}{$(111)$} \\
\hline Photon energy (keV) & 12.6 & \multicolumn{2}{|c|}{12.6} \\
\hline Bragg angle $\left({ }^{\circ}\right)$ & 13.8 & \multicolumn{2}{|c|}{8.99} \\
\hline Monochromator size $(\mathrm{cm} \mathrm{x} \mathrm{cm)}$ & $4.7 \times 2.2$ & \multicolumn{2}{|c|}{$6.6 \times 2.2$} \\
\hline Beam footprint, $\mathrm{v} \times \mathrm{h}(\mathrm{cm} \times \mathrm{cm})$ & $0.75 \times 0.36$ & \multicolumn{2}{|c|}{$1.2 \times 0.36$} \\
\hline Diffracting element size $(\mathrm{cm} \times \mathrm{cm})$ & $0.75 \times 0.36$ & \multicolumn{2}{|c|}{ NA } \\
\hline Diffracting element thickness $(\mathrm{cm})$ & 0.05 & \multirow{2}{*}{\multicolumn{2}{|c|}{$\begin{array}{l}\text { NA } \\
23\end{array}$}} \\
\hline Peak incident heat flux $\left(\mathrm{W} / \mathrm{mm}^{2}\right)$ & 35 & & \\
\hline Monochromator substrate thickness $(\mathrm{cm})$ & 1.0 & 1.0 & 0.1 \\
\hline Max. temperature rise abo:e gallium temperature $\left({ }^{\circ} \mathrm{C}\right)$ & 55 & 660 & 506 \\
\hline Max. temp. rise at wall-Ga interface $\left({ }^{\circ} \mathrm{C}\right)$ & 18 & 27 & 280 \\
\hline Max. temp. rise across the cystal $\left({ }^{\circ} \mathrm{C}\right)$ & 37 & 633 & 226 \\
\hline Max. compressive stress ( $\mathrm{M}$ a) & 21 & 154 & 136 \\
\hline Max. tensile stress (MPa) & 8 & 46 & 33 \\
\hline Max. displacement in the scattering plane $(\mu \mathrm{m})$ & 0.27 & 14 & 18 \\
\hline Max. slope error in the scattering plane (arc second) & 5 & 180 & 200 \\
\hline
\end{tabular}

The slope errors along the AA'-axis for the two 1 -cm thick monochromators are shown in Fig. 8, where again, the footprint regions are highlighted. The maximum slope errors, which occur near periphery of the footprints, are about 5 arc second for diamond and 180 arc seconc.s for silicon. Since the photon beams have typically Gaussian profiles with their peaks where slope error is negligible and their nadir where the slope error is maximum, the effective slope errors are somewhat smaller.

\section{SUMMARY AND CONCLUSIONS}

We have proposed a diamond-based monochromator for very high heat load beamlines. The monochromator consists of a small single crystal diamond bonded to a polycrystalline diamond substrate in which the necessary cooling channels are configured.

A preliminary study of the subject examines the potential of such a monochromator. From a thermalstructural point of view, a diamond moi: - hromator is vastly superior to a silicon monochromator. A simulated comparison of a silicon versus a cusinond monochromator subjected to the APS Undulator A beam at closed gap indicates slope errors of 180 and 5 arc seconds, respectively. While the inclined monochromator 2,3 provides a solution to the high heat load monochromator problem, a diamond monochromator allows conventional (non-inclined) operation of a monochromator. in addition, a diamond monochromator can be devised to operate in an inclined mode, in which case there is potential to 
be able to handle heat fluxes an order of magnitude higher than those generated by undulators in the near future. This last statement assumes that one can obtain larger $(2-4 \mathrm{~cm}$ size) single crystal diamonds.

Preliminary lesting of Sumitomo synthetic single crystal diamonds, $5 \mathrm{~mm}$ x $5 \mathrm{~mm} \times 0.3 \mathrm{~mm}$, gave a double crystal rocking curve (for Mo K-LIII) of about 6 arc second compared to the theoretical value of about 1 are second. These measurements must be carelully repeated. Further study and characterization of these crystals is necessary to evaluate their suitability as monochromator material. ${ }^{24}$ It should also be realized that, unlike silicon, commercially available diamonds are restricted to a limited number of crystal orientations.

A number of additional issues must be investigated to determine the suitability of diamond as the monochromator material for routine use on high heat load synchrotron beamlines. 25 These include (a) strain-free bonding of diamond to diamond, or deposition of CVD diamond on the single crystal diffracting element, (b) dimensional stability of a composite diamond monochromator, (c) radiation damage study for diamond, $26-28$ and (d) further investigation of the diffraction properties of commercially available diamond crystals over large areas.

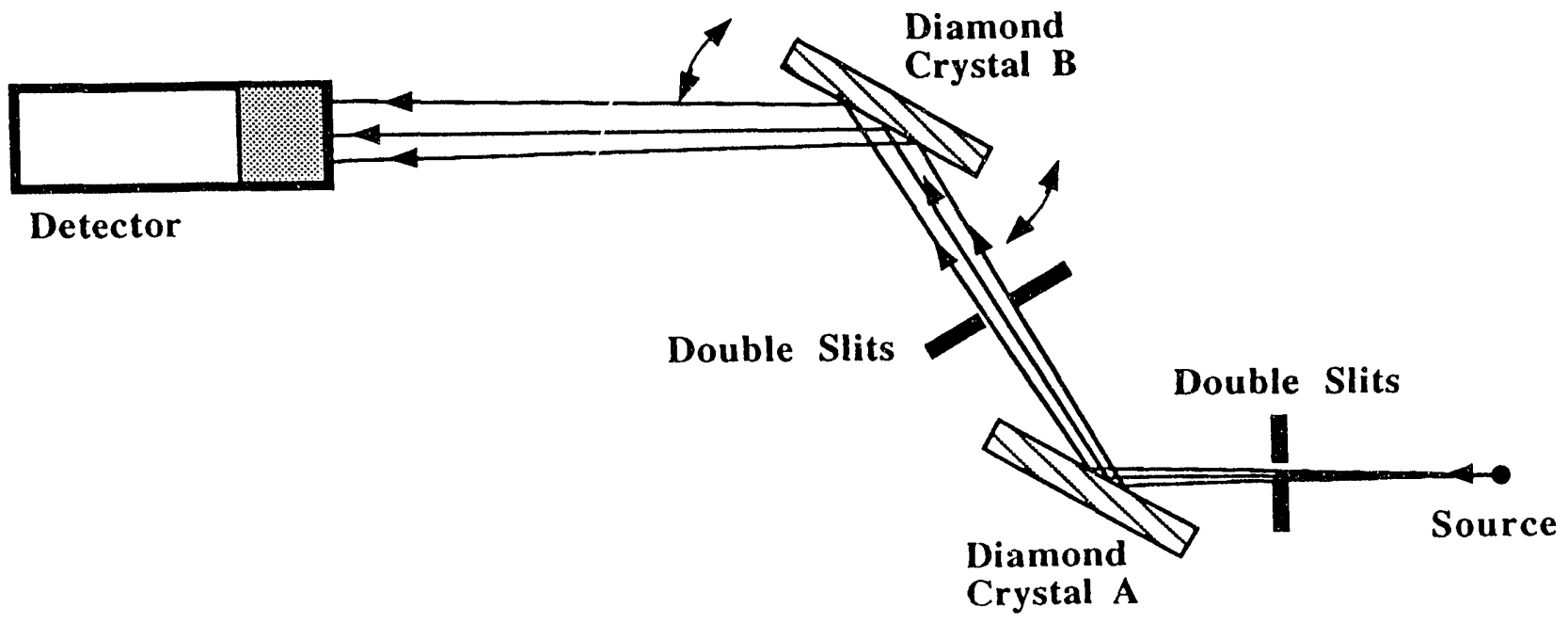

Figure 1. Experimental setup (viewed from the top) for the rocking curve measurements. The $x$-ray beam from the Mo x-ray source on the right passes through a set of double slits and is incident on the Crystal A. Crystal A diffracts the K-LIII $\mathrm{x}$-ray line, which passes through the serond set of double slits and is diffracted a second time by the Crystal $\mathrm{B}$. The final intensity is detected in the detector on the left. 


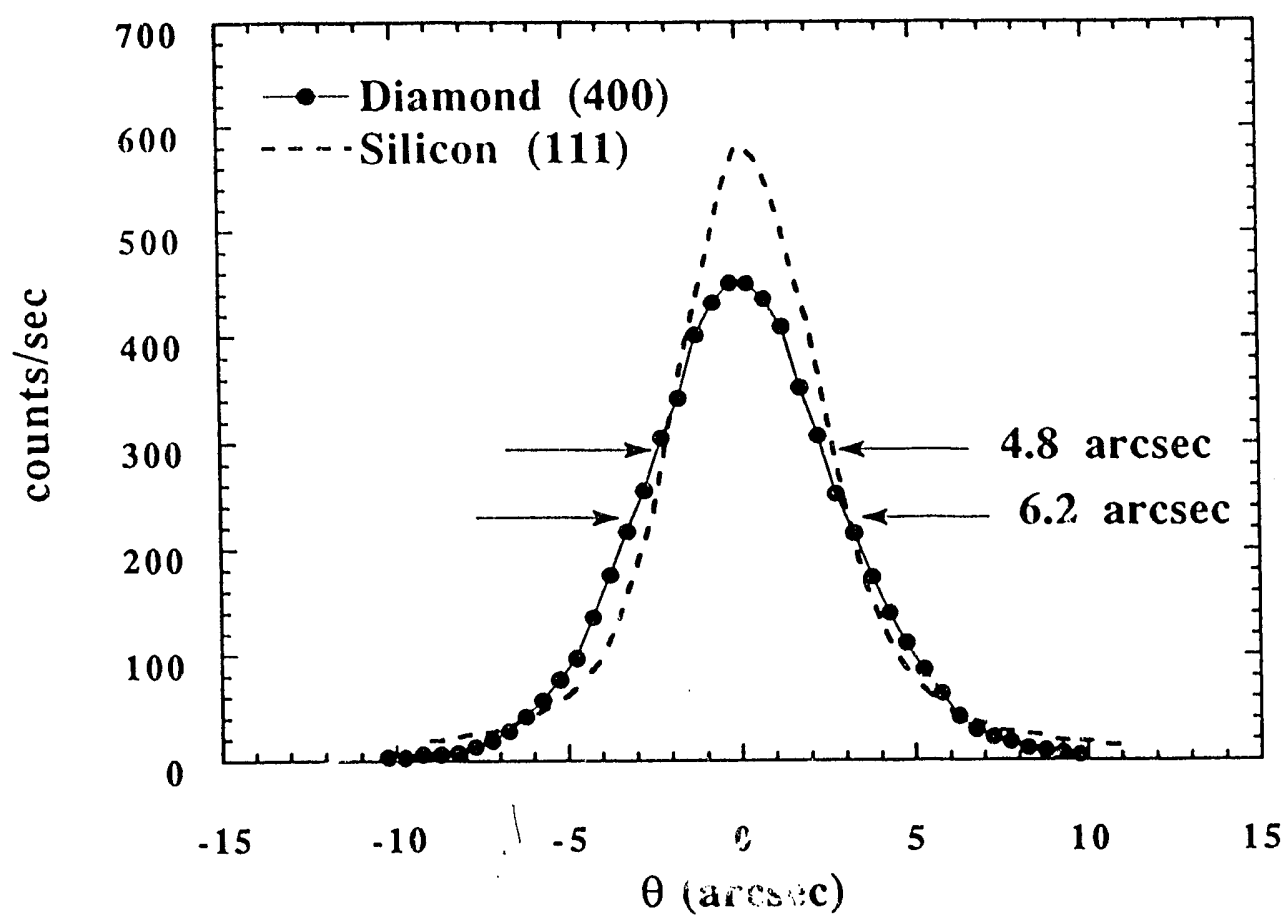

Figure 2. Double crystal rocking curves for the synthetic diamond (400) and silicon (111) crystals with the Mo K-LIII $x$-ray. The counting rate in the detector is plotted versus the Bragg diffraction angle of the second crystal using an arbitrary zero located near the center of the peak.

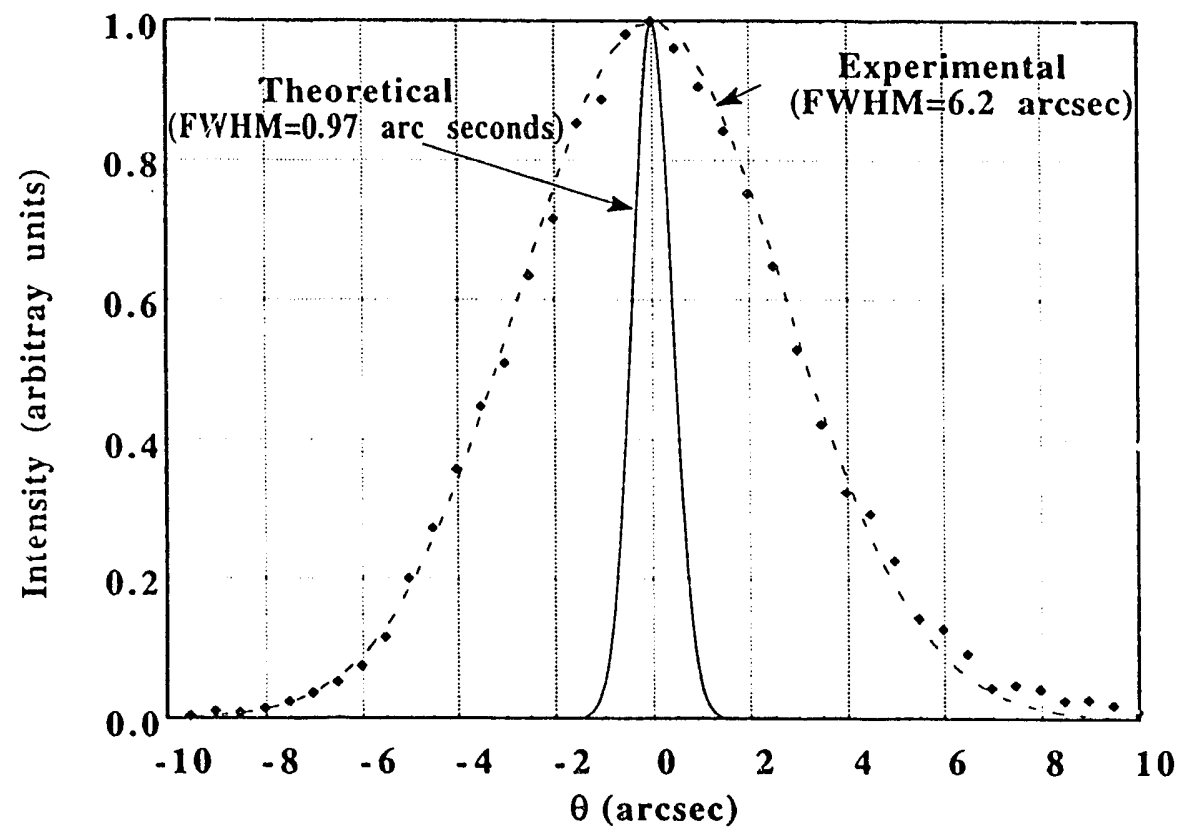

Figure 3. Comparison of the measured and theoretical double crystal rocking curves of diamond (400) using photons from Mo K-LIII. 


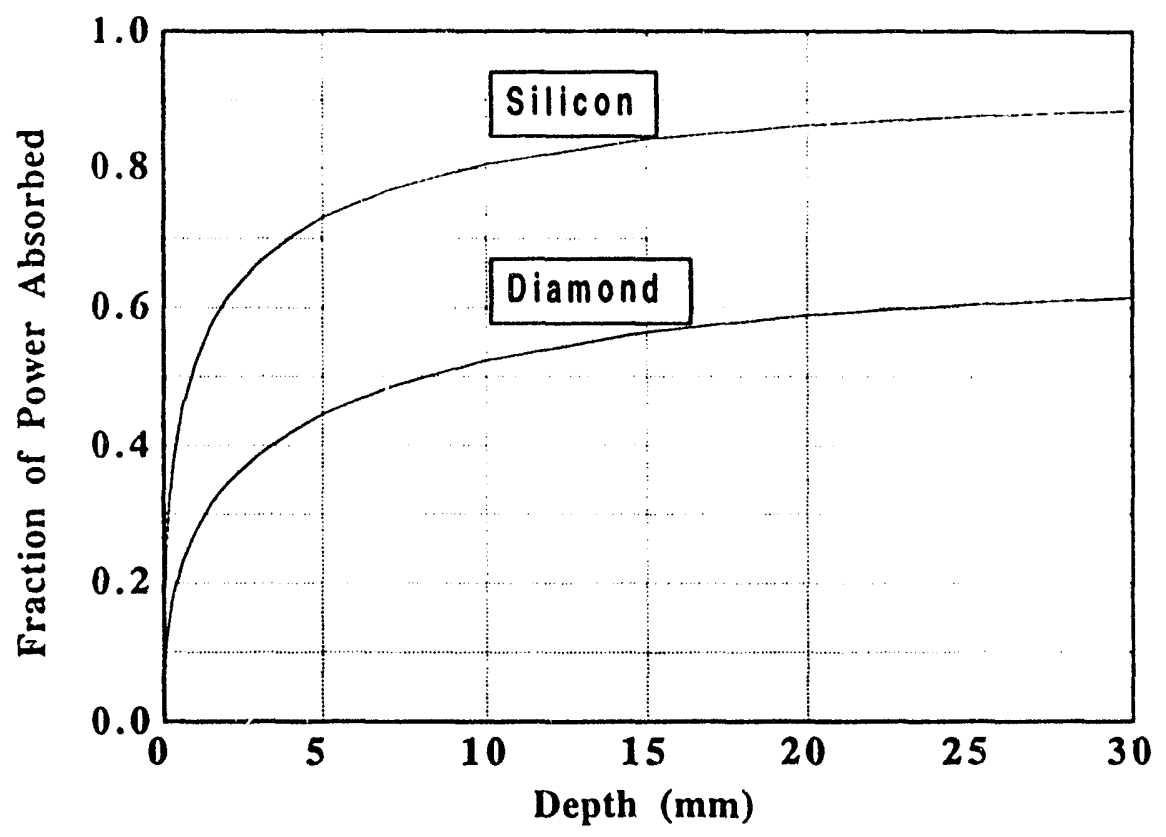

Figur the absorption of the APS Undulator A beam in diamond and silicon computed from a bending magnet approximation for the source with a characteristic energy of $23.5 \mathrm{keV}$.

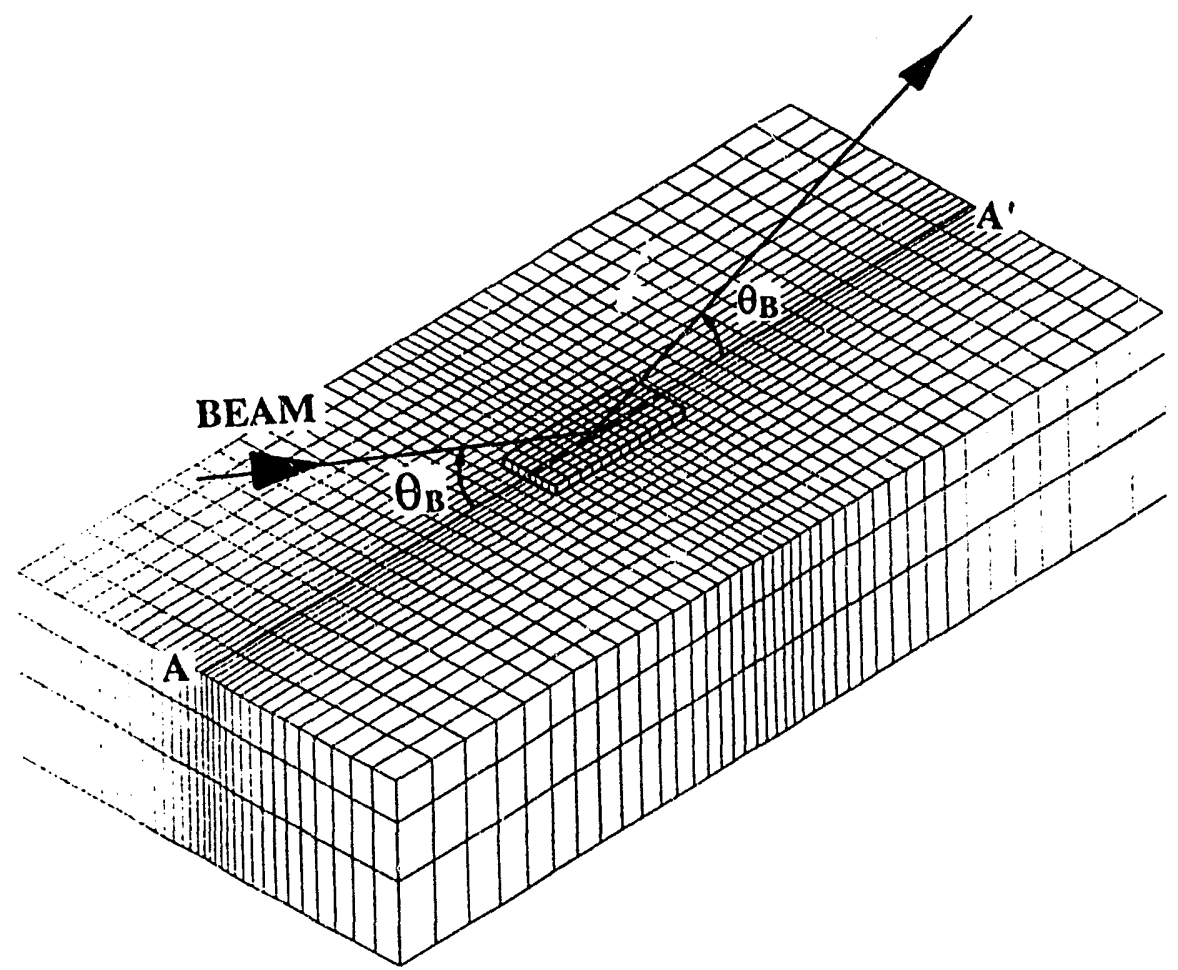

Figure 5. The monochromator model used in the analysis of the diamond monochronnator. The substrate is CVD diamond while the diffracting element bonded to it is single crystal diamond shown in heavy lines. 


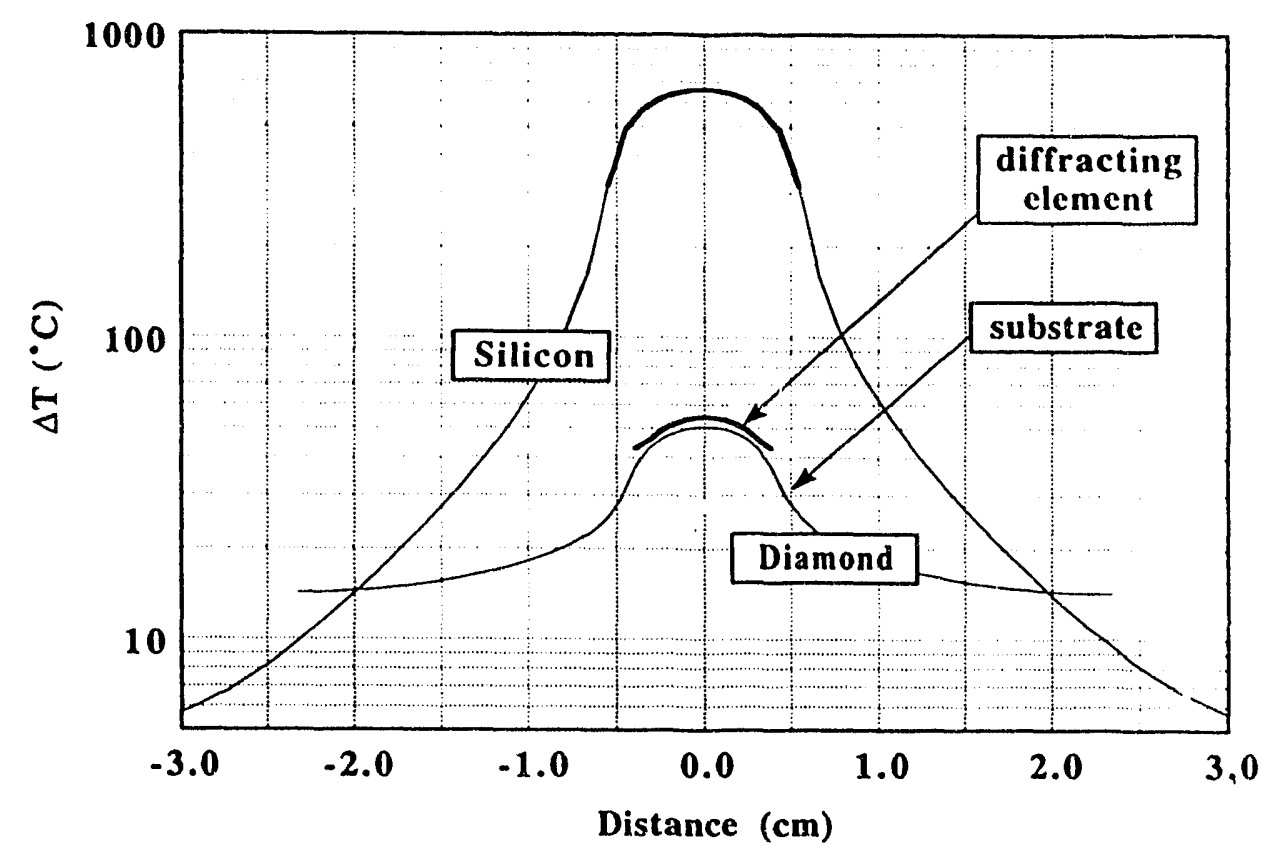

Figure 6. Temperature rise in the 1-cm-thick silicon and diamond crystals along the AA'-axis of Fig. 5.

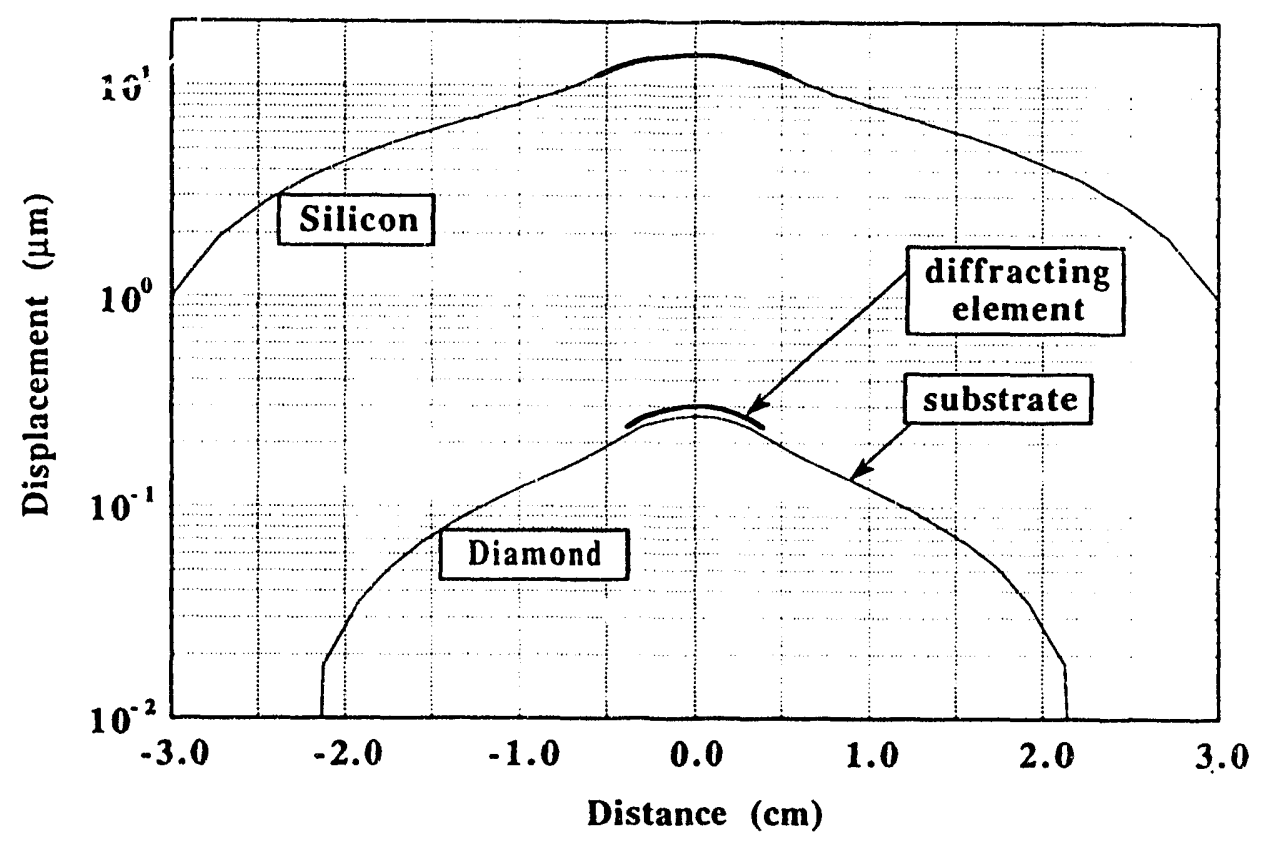

Figure 7. The displacement along the $\mathrm{AA}^{\prime}$-axis of Figure 5 in the $1-\mathrm{cm}$-thick silicon and diamond crystals under the APS undulator beam at closed gap. 


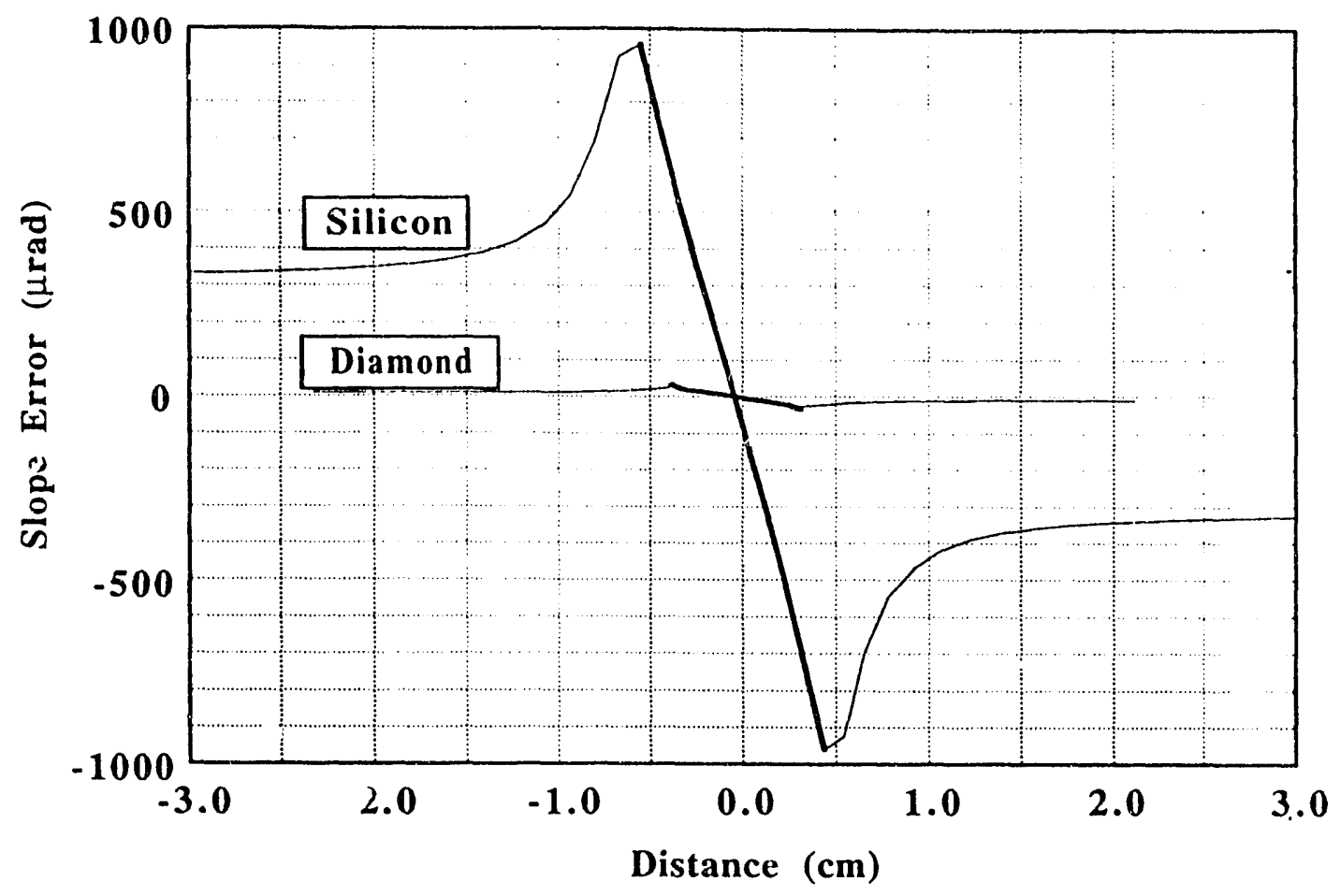

Figure 8. Slope errors along the AA'-axis of Figure 5 for the silicon and diamond crystals under the APS Undulator beam at closed gap.

\section{ACKNOWLEDGMENTS}

This work was supported in part by the U. S. Department of Energy BES Materials Science under Contract No. W-31-109-ENG-38. We would like to thank Den:is Mills for his helpful comments and David Lunt for obtaining surface profiles of the synthetic diamond crystals. The crystals were kindly provided by Mr. I. Nakamura of Sumitomo Electric USA. We would like to thank Patricia Fernandez for her assistant in rocking curve measurements and S. Picologlou for editing this manuscript.

\section{REFERENCES}

1. R.K Smither and A.K. Freund, editors, Workshop on High Heat Load X-Ray Optics, Workshop Report No. ANL/APS/TM-6, Advanced Photon Source, Argonne National Laboratory, Argonne, Illinois, USA, 1989.

2. A.M. Khounsary, "A Novel Monochromator for High Heat Load Synchrotron X-Ray Radiation," Rev. Sci. Instrum. 63, 461-464, 1992.

3. A.T. Macrander, W.K. Lee, R.K. Smither, and D.M. Mills, C.S. Rogers, and A.M. Khounsary, "High Heat Load Performance of an Inclined Crystal Monochromator with Liquid Gallium Cooling on the CHESS-ANL Undulator,", Nucl. Instrum. Meth. A319, 181-196, 1992.

4. R.C. Evans, P.B. Hirsch, and J.N. Keller, " A Parallel Beam Concentrating Monochromator for X- 'ays," Acta Cryst. 1, pp. 124-129, 1948. 
5. Y.S. Touloukian and E.H. Buyco, editors, Thermophysical Properties of Matter, IFI/Plenum Pub., New York, 1970.

6. R. Berman, Physical Properties of Diamond, Clarendon Press, Oxford, 1965.

7. J. Wilks and E. Wilks, Properties and Applications of Diamond, Butterworth Heinemann, 1991.

8. J.E. Field, editor, The Properties of Diamond Academic Press, 1979.

9. J.T. Glass, R. Messier, and N. Fujimori, editors, Diamond, Silicon, Carbide, and Related Wide Bandgap Semiconductors, 1990.

10. Dubbeldec Harris Diamond Corp., $M \cdots n$ Arlington, NJ 07856, private communication witi AMK, 1992.

11. M. Seal, Amsterdam, Holland, private conversation with AMK, 1992.

12. P.L. Hanley, I. Kiflawi, and A.R. Lang, "On topographically identifiable sources of cathodoluminescence in natural diamond," Phil. Trans. R. Soc. Lond. A.284, 329-363, 1977.

13. A. Jackson, "The NINA polarised photon beam," Nicl. Instrum. and Meth., 129, 73-83, 1975.

14. S. Bernnan, and P.L. Cowan, "A suite of FORTRAN programs for calculating perfect crystal $x$ ray diffraction performance for arbitrary crystals at arbitrary wavelength," Rev. Sci. Instrum., 63, 850$853,1992$.

15. Sumitomo Electric USA, Inc., Los Angeles, CA, private communications with AMK, 19901992.

16 H.P. Bovenkerk, F.P. Bundy, H.T. Hall, H.H. Strong, and R.H. Wentrof, "Preparation of diamond," Nature, 184, 1094-1098, 1959.

17. R. Diangello, General Electric, Schenectady, NY, Private communications with AMK, 1992.

18. L. Berman, Brookhaven National Laboratory, NY, Private communication with AMK, 1992.

19. H. Holloway, K.C. Hass, and M.A. Tamor, T.R. Anthony, and W.F. Banhoizer, "Isotropic dependence of the lattice constant of diamond," Phys. Rev. B, 44, 7123-7126, 1991.

20. G.K.. Shenoy, P. J. Viccaro, and D. M. Mills, "Characteristics of the 7-GeV Advanced Photon Source: A Gu:de for Users, "Argonne National Laboratory Report ANL-88-9, Feb. 1988.

21. D. Chapman, N. Gmuer, N. Lazarz, and W. Thomlinson, "PHOTON: A program for synchrotron radiation dose calculations," Nucl. Instrum. and Meth. A266, 191-194, 1988.

22. J.E. Graebner, S. Lin, G.W. Kammlott, J.A. Herb, and C.F. Gardinier, "Unusually high thermal conductivity in diamond films," Appl. Phys. Lett. 60, 1576-1578, 1992.

23. M. Seal, "A review of methods of bonding or making electrical contacts to diamond," Diamond Review 29, 408, 1969.

24. B.K. Tanner, X-Ray Diffraction Topography, Pergamon Press, 1976

25. A.M. Khounsary and T.M. Kuzay, "On diamond windows for high power synchrotron $x$-ray beams," Nucl. Instrum. Meth. A319, 233-239, 1992.

26. M. Itoh, M. Hori, H. Komano, and I. Mori, "A study of radiation damage in SiN and SiC mask membranes," J. Vac. Sci. Technol B9, 3262-3265 1991.

27. Suzuki, R. Kumar, H. Windischmann, H. Sano, Y. Iimura, H. Miyashita, and N. Watanabe, "X-ray irradiation effects on a microwave-plasma chemical vapor deposition diamond membrane, "J. Vac. Sci. Technol. B9, 3266-3269, 1991.

28. G.M. Wells, S. Palmur, and F. Cerrina, A. Purdes, and B. Gnade, J. Vac. Sci. Technol. "Radiation stability of $\mathrm{SiC}$ in diamond membranes as potential $\mathrm{x}$-ray lithography mask carriers," B8, 1575-1578, 1990. 

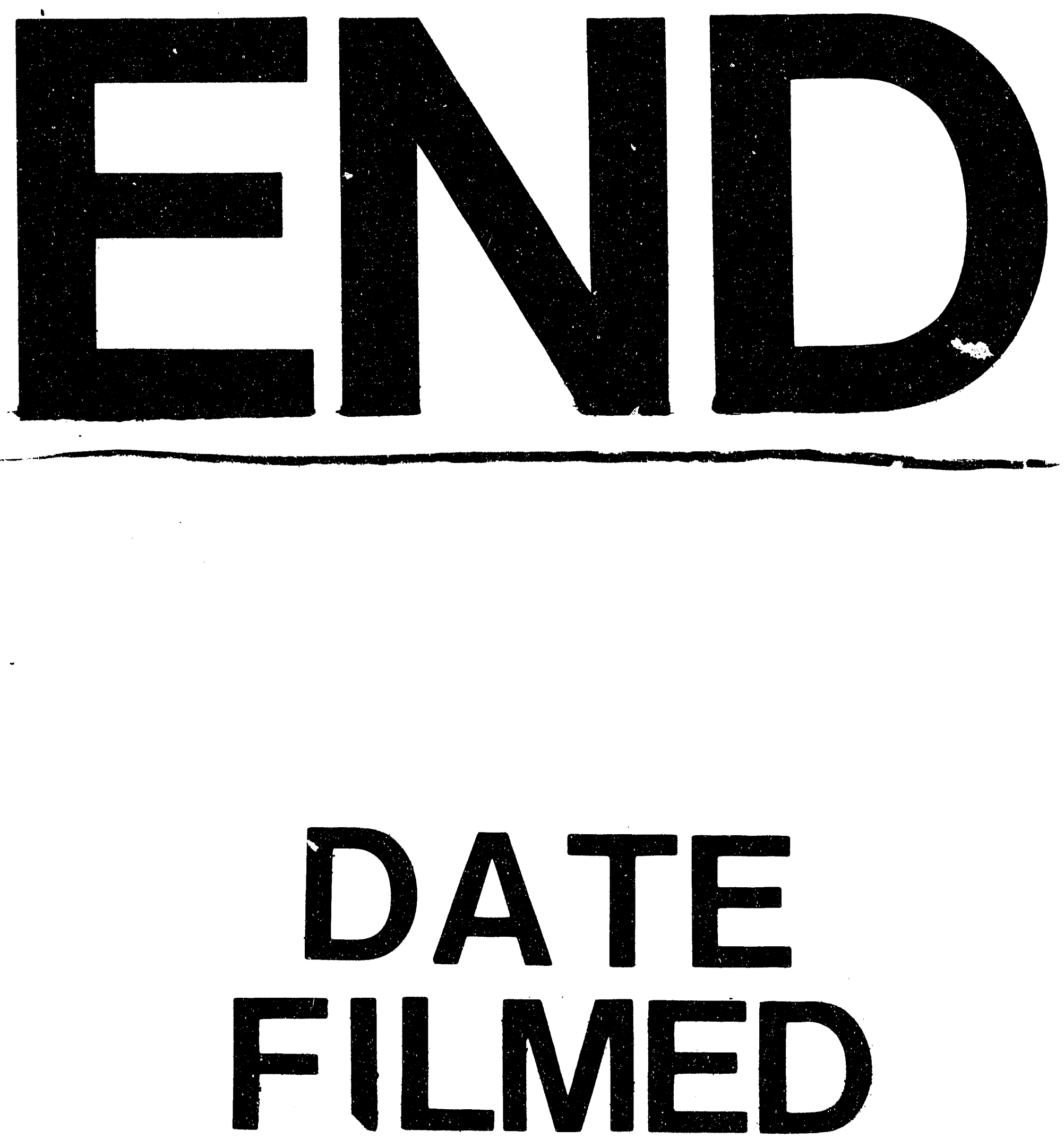

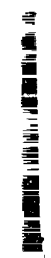

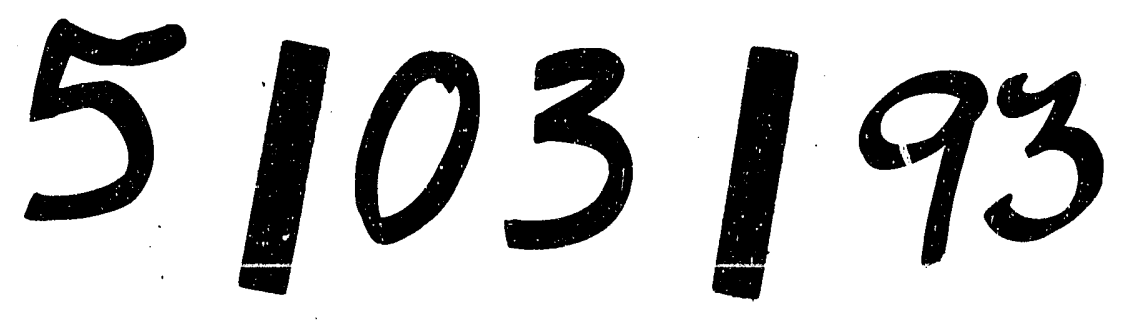


1 